\title{
TOEPLITZ OPERATORS WITH RADIAL SYMBOLS ON BERGMAN SPACE AND SCHATTEN-VON NEUMANN CLASSES
}

\author{
Z. BENDAOUD, S. KUPIN, K. TOUMACHE, B. TOURÉ, AND R. ZAROUF
}

\begin{abstract}
In the present paper, we study spectral properties of Toeplitz operators with (quasi-) radial symbols on Bergman space. More precisely, the problem we are interested in is to understand when a given Toeplitz operator belongs to a Schatten-von Neumann class. The methods of the approximation theory (i.e., Legendre polynomials) are used to advance in this direction.
\end{abstract}

\section{INTRODUCTION}

The study of Toeplitz operators is an important topic of the modern operator theory. In forties-eighties of the last century, the research was focused on Toeplitz operators on Hardy spaces, see Nikolski [16] for a complete account of results on the topic and the references. Since mid-eighties, the interest shifted to operators defined on different spaces of analytic functions, such as Bergman, Fock and other spaces. We quote the articles by Axler [4], Luecking [14, 15], Zhu [22] and later papers by Grudsky-Vasilevski [8], Grudsky-Karapetyants-Vasilevski [9], Stroethoff [19], Korenblum-Zhu [10] and Zorboska [20]. These articles focused on the study of the analytical features of a Toeplitz operator (i.e., its boundedness, compactness, etc.) related to the properties of its symbol, see (0.1) for the definitions, and they serve a basis for our approach.

In the present article, we pursue in this direction and we give a series of sufficient conditions for Toeplitz operators with radial complex symbols to belong to the so called Schatten-von Neumann classes. In particular, we suppose the symbols neither real nor sign-definite. What seems to be more important is that we suggest a method of solving the direct and inverse spectral problems for operators of this class. It relies on the techniques of approximation theory and it makes an extensive use of Legendre orthogonal polynomials on an interval.

To make all this more precise, we introduce the notation and give some definitions. Let $\mathbb{D}=\{z:|z|<1\}$ be the unit disk, $\mathbb{T}=\{z:|z|=1\}$ the unit circle and

$$
d A(z)=\frac{1}{\pi} d x d y
$$

be the probability area measure on $\mathbb{D}$. For $1 \leq p \leq \infty$, consider

$$
L^{p}(\mathbb{D})=L^{p}(\mathbb{D}, d A)=\left\{f:\|f\|_{p}^{p}=\int_{\mathbb{D}}|f(z)|^{p} d A(z)<+\infty\right\},
$$

and the corresponding subspaces of analytic functions

$$
L_{a}^{p}(\mathbb{D})=L_{a}^{p}(\mathbb{D}, d A)=\left\{f: f \text { is analytic on } \mathbb{D},\|f\|_{p}^{p}=\int_{\mathbb{D}}|f(z)|^{p} d A(z)<+\infty\right\} .
$$

These spaces $L_{a}^{p}(\mathbb{D})$ are called Bergman spaces, see Hedenmalm-Korenblum-Zhu [6] and Zhu [21] for more details on the topic.

Date: February, 15, 2018

2010 Mathematics Subject Classification. Primary: 47B35; Secondary: 30H20, 42C10. 
We are mainly concerned with the Hilbert case $p=2$. As usual, the scalar product in $L^{2}(\mathbb{D})$ is defined as

$$
(f, g)=\int_{\mathbb{D}} f(z) \bar{g}(z) d A(z), \quad f, g \in L^{2}(\mathbb{D}) .
$$

It is well known that there is a bounded orthogonal projection (called Bergman projection) $P_{+}: L^{2}(\mathbb{D}) \rightarrow L_{a}^{2}(\mathbb{D})$. This projecton admits the integral representation

$$
\left(P_{+} f\right)(w)=\int_{\mathbb{D}} \frac{f(z)}{(1-z \bar{w})^{2}} d A(z), \quad f \in L^{2}(\mathbb{D}),
$$

see [6, Sect. 1.1].

It is easy to see that polynomials with respect to $z$ (denoted by $\mathcal{P}$ ), form a dense subset of $L_{a}^{2}(\mathbb{D})$. Now, let $\varphi \in L^{2}(\mathbb{D})$ and define the Toeplitz operator with symbol $\varphi$ as

$$
T_{\varphi}: L_{a}^{2}(\mathbb{D}) \rightarrow L_{a}^{2}(\mathbb{D}), \quad T_{\varphi} p=P_{+}(\varphi p), \quad p \in \mathcal{P} .
$$

We say that the operator $T_{\varphi}$ is bounded, if one can extend it by continuity to the whole $L_{a}^{2}(\mathbb{D})$ and the resulting operator is still bounded. We denote the extended operator by $T_{\varphi}$ as well.

The class of compact operators is denoted by $\mathcal{S}_{\infty}$. The Schatten-von Neumann classes are defined as

$$
\mathcal{S}_{p}=\left\{A \in \mathcal{S}_{\infty}:\|A\|_{\mathcal{S}_{p}}^{p}=\sum_{k} s_{k}(A)^{p}<\infty\right\}, 0<p<\infty,
$$

where $\left\{s_{k}(A)\right\}$ are the singular numbers of the operator $A$. We refer to Simon [18], Gohberg-Goldberg-Kaashoek [7] for more information on the subject.

Write $z=|z| e^{i \theta}=r e^{i \theta} \in \mathbb{D}$. One says that the symbol $\varphi$ is radial (or homogeneous), if $\varphi(z)=\varphi(|z|)=\varphi(r)$, i.e., $\varphi$ is rotationally symmetric with respect to the origin. With a slight abuse of notation, we consider a radial symbol $\varphi$ as a function on $\mathbb{D}$ and, keeping the same letter, as a function on $[0,1)$. When needed, we extend it to a function defined on $[0,1]$ letting $\varphi(1)=0$. One says that the symbol is quasi-radial (or quasi-homogeneous), if $\varphi(z)=\varphi(|z|) e^{i m \theta}$ for some fixed $m \in \mathbb{Z}$.

As we already mentioned, we wish to understand when a Toeplitz operator $T_{\varphi}$ with a radial symbol lies in the Schatten-von Neumann class $\mathcal{S}_{p}, 0<p<\infty(0.2)$. We pay a particular attention to the study of complex-valued or sign-indefinite symbols. The case of quasi-homogeneous symbols follows similarly. A sample of known results in this direction is given in Subsection 1.1.

The spectrum of a Toeplitz operator of the above type is rather easy to compute. Indeed, taking the standard orthogonal basis $e_{n}(z)=\sqrt{n+1} z^{n}$ for $L_{a}^{2}(\mathbb{D})$, one sees that the matrix elements $\left(T_{\varphi}\right)_{n m}=\left(T_{\varphi} e_{n}, e_{m}\right)$ are given by $(1.1)$, and so the spectrum $\sigma\left(T_{\varphi}\right)$ is given by $\overline{\left\{c_{n}(\varphi)\right\}}$, where

$$
c_{n}(\varphi):=\left(T_{\varphi}\right)_{n n}=2(n+1) \int_{0}^{1} x^{2 n+1} \varphi(x) d x .
$$

We sketch now the idea on the construction of the spectral maps for radial Toeplitz operators, see Subsections 3.1, 3.2 for more details. To this end, we consider the Legendre polynomials, i.e., the polynomials which are orthogonal in $L^{2}[-1,1]=L^{2}([-1,1], d s)$, see also Subsection 1.2.

Let $\varphi \in L^{2}([0,1], x d x)$ be the symbol of a radial Toeplitz operator. We set $\psi(s)=\varphi(\sqrt{s}), s \in[0,1]$ and

$$
\psi_{e}(s)=\left\{\begin{array}{ll}
\psi(x), & x \in[0,1], \\
\psi(-x), & x \in[-1,0],
\end{array} \quad \psi_{o}(s)= \begin{cases}\psi(x), & x \in[0,1], \\
-\psi(-x), & x \in[-1,0) .\end{cases}\right.
$$


It is plain that $\psi_{e}, \psi_{o} \in L^{2}([-1,1], d s)$ and we can write

$$
\psi_{e}(s)=\sum_{k=0}^{\infty} a_{2 k} P_{2 k}(s), \quad \psi_{o}(s)=\sum_{k=0}^{\infty} a_{2 k+1} P_{2 k+1}(s),
$$

where

$$
\begin{aligned}
a_{2 k} & =\frac{1}{\left\|P_{2 k}\right\|_{L^{2}[-1,1]}^{2}}\left(\psi_{e}, P_{2 k}\right)_{L^{2}[-1,1]}=\frac{(4 k+1)}{2}\left(\psi_{e}, P_{2 k}\right)_{L^{2}[-1,1]}, \\
a_{2 k+1} & =\frac{1}{\left\|P_{2 k+1}\right\|_{L^{2}[-1,1]}^{2}}\left(\psi_{0}, P_{2 k+1}\right)_{L^{2}[-1,1]}=\frac{(4 k+3)}{2}\left(\psi_{0}, P_{2 k+1}\right)_{L^{2}[-1,1]} .
\end{aligned}
$$

Above, $\|\cdot\|_{L^{2}[-1,1]}$ and $(., .)_{L^{2}[-1,1]}$ stay for the usual norm and the scalar product in $L^{2}[-1,1]$, respectively. We have that

$$
\left\{c_{n}(\varphi)\right\}=\mathcal{D}\left(\left\{a_{k}\right\}\right), \quad\left\{a_{k}\right\}=\mathcal{I}\left(\left\{c_{n}(\varphi)\right\}\right),
$$

where the maps $\mathcal{D}$ and $\mathcal{I}$ are given by (3.5), (3.6), see (3.2), (3.4) for the definitions of the corresponding coefficients.

As an application of this construction, we prove the following theorem.

Theorem (= Theorem 3.3). Take an arbitrary $\delta_{0}>0$. There exists $a \varphi \in$ $L^{2}([0,1], x d x)$ such that

(1) $\varphi^{\prime} \notin L^{2}([0,1], x d x)$,

(2) Toeplitz operator $T_{\varphi}(0.1)$ with radial symbol $\varphi$ lies in $\mathcal{S}_{\delta_{0}}$.

Further results of this type are in progress and they are to be published elsewhere. Notice that this theorem gives a different point of view on results of Propositions $2.1,2.3$, and the related examples.

The article is organized as follows. The first part of Section 1 contains a reminder on notions related to Bergman spaces and basic computations on radial Toeplitz operators there. To put the things in perspective, we give a brief account of known results on the topic as well. The second part of Section 1 gives a summary of facts on Legendre polynomials. Section 2 presents certain sufficient conditions for radial Toeplitz operators to lie in the classes $\mathcal{S}_{p}, 0<p<\infty$ and a related example. The first part of Section 3 explains the construction of the spectral maps (3.5), (3.6). Its second part deals with the proof of Theorem 3.3.

In this paper, $C$ stays for a constant changing from one relation to another; we give usually the list of parameters the constant depends on.

\section{Preliminaries}

1.1. Notions related to Bergman spaces and some results on Toeplitz operators. An extensive account on Bergman spaces can be found in HedenmalmKorenblum-Zhu [6] and Zhu [21].

Recall the spaces $L^{p}(\mathbb{D})$ and $L_{a}^{p}(\mathbb{D}), 1 \leq p \leq \infty$, defined in the previous section. An important property of the space $L_{a}^{2}(\mathbb{D})$ is that for any $w \in \mathbb{D}$, there exists the so called reproducing kernel, i.e., $k_{w} \in L_{a}^{2}(\mathbb{D})$ given by

$$
k(z, w)=k_{w}(z)=\frac{\left(1-|w|^{2}\right)}{(1-\bar{w} z)^{2}}, \quad z, w \in \mathbb{D} .
$$

One has that for any $f \in L_{a}^{2}(\mathbb{D})$

$$
\left(1-|w|^{2}\right) f(w)=\left(f, k_{w}\right),
$$

where $(.,$.$) is the scalar product in L^{2}(\mathbb{D})$. For more information on the reproducing kernels, see [6, Sect. 1.1], [21, Ch. 4]. 
For a function $g \in L^{1}(\mathbb{D})$, one considers its Berezin transform [6, Ch. 2], [21, Ch. 6] defined as

$$
\tilde{g}(w)=\left(g k_{w}, k_{w}\right), \quad w \in \mathbb{D} .
$$

Similarly, for a Toeplitz operator $T_{\varphi}(0.1)$, one sets

$$
\tilde{T}_{\varphi}(w)=\tilde{\varphi}(w)=\left(T_{\varphi} k_{w}, k_{w}\right)=\left(P_{+} \varphi k_{w}, k_{w}\right)=\left(\varphi k_{w}, k_{w}\right), \quad w \in \mathbb{D} .
$$

As we will see, many properties of $T_{\varphi}$ can be expressed conveniently in terms of $\tilde{\varphi}$.

The next two results hold for general Toeplitz operators $T_{\varphi}$ (i.e., , the symbols $\varphi$ are not required to be radial). Let $\beta(z, w), z, w \in \mathbb{D}$, be the Bergman (or, equivalently, hyperbolic) metric on $\mathbb{D}$, see [21, Ch. 4], and

$$
D(w, r)=\{z \in \mathbb{D}: \beta(z, w)<r\},
$$

where $r \geq 0$. For a fixed $r$, define the averaging of $\varphi$ as

$$
\hat{\varphi}_{r}(w)=\frac{1}{A(D(w, r))} \int_{D(w, r)} \varphi(z) d A(z),
$$

where $A(D(w, r))=\int_{D(w, r)} d A(z)$.

Theorem 1.1 ([21, Ch. 9]).

(1) (see $[21$, Ch. 7, Prop. 7.3]) Let $\varphi \in C(\overline{\mathbb{D}})$ (i.e., $\varphi$ is continuous on $\overline{\mathbb{D}})$. Then $T_{\varphi}$ is compact (i.e., $T_{\varphi} \in \mathcal{S}_{\infty}$ ) iff

$$
\lim _{|z| \rightarrow 1-} \varphi(z)=0 \text {. }
$$

(2) (see [21, Ch. 7, Cor. 7.9]) Let $\varphi(z) \geq 0$ on $\mathbb{D}$. Then the following is equivalent:

- $T_{\varphi} \in \mathcal{S}_{\infty}$,

- $\lim _{|z| \rightarrow 1-} \tilde{\varphi}(z)=0$,

- for any fixed $r>0, \lim _{|z| \rightarrow 1-} \hat{\varphi}_{r}(z)=0$.

Put also $d \lambda(z)=d A(z) /\left(1-|z|^{2}\right)^{2}$.

Theorem 1.2 ([21, Ch. 7]). Let $\varphi(z) \geq 0$ and $p \geq 1$. Then the following is equivalent:

- $T_{\varphi} \in \mathcal{S}_{p}$,

- $\tilde{\varphi} \in L^{p}(\mathbb{D}, d \lambda)$,

- for any fixed $r>0, \hat{\varphi}_{r} \in L^{p}(\mathbb{D}, d \lambda)$.

From now on, we suppose the symbol $\varphi$ to be radial. Then the matrix of the Toeplitz operator $T_{\varphi}$ is simple to compute. Taking the standard basis $e_{n}(z)=$ $\sqrt{n+1} z^{n}$ of $L_{a}^{2}(\mathbb{D})$, one has

$$
\left(T_{\varphi}\right)_{n m}=\frac{(n+1)^{1 / 2}(m+1)^{1 / 2}}{\pi} \int_{0}^{2 \pi} e^{i \theta(n-m)} d \theta \int_{0}^{1} r^{n+m+1} \varphi(r) d r .
$$

So

$$
\left(T_{\varphi}\right)_{n m}= \begin{cases}2(n+1) \int_{0}^{1} r^{2 n+1} \varphi(r) d r, & m=n \\ 0, & n \neq m\end{cases}
$$

and the matrix of the operator is diagonal. It is convenient to set

$$
c_{n}(\varphi):=\left(T_{\varphi}\right)_{n n}=2(n+1) \int_{0}^{1} r^{2 n+1} \varphi(r) d r .
$$

For a radial symbol $\varphi$, its Berezin transform is also radial and it is computed as

$$
\tilde{\varphi}(z)=\left(1-|z|^{2}\right)^{2} \sum_{n=0}^{\infty}(n+1) c_{n}(\varphi)|z|^{2 n},
$$


see Zobroska [20].

Notice that one does not suppose $\varphi$ to be sign-definite in the following theorem.

Theorem 1.3 (Korenblum-Zhu [10]). Let $T_{\varphi}$ be a Toeplitz operator with a radial bounded symbol $\varphi$. Then the following is equivalent:

(1) $T_{\varphi} \in \mathcal{S}_{\infty}$

(2) $\lim _{n \rightarrow+\infty} 2(n+1) \int_{0}^{1} \varphi(x) x^{2 n+1} d x=0$.

(3) $\lim _{t \rightarrow 1-} \frac{1}{1-t} \int_{t}^{1} \varphi(x) d x=0$.

We finish this subsection mentioning interesting related papers by LouhichiZakariasy [11], Louhichi-Strouse-Zakariasy [12], and Louhichi-Randrimahaleo-Zakariasy [13].

1.2. Few facts on Legendre polynomials. To advance on the problem presented in the Introduction, the idea is to use a "natural" basis of $L^{2}[-1,1]=$ $L^{2}([-1,1], d x)$. Of course, the system $\left(x^{n}\right)_{n \in \mathbb{N}}$ does not suit this purpose, since it is complete, but, as we will see, it is very far from an orthogonal (or Riesz) basis.

A much better choice are the so called orthogonal Legendre polynomials $\left\{P_{n}(x)\right\}$. Below, we give a short list of their properties needed for our construction presented in Section 3. For extensive information on the subject, see the books by ErdélyiMagnus-Oberhettinger et al. [5, Ch. 3] and Arfken-Weber [3, Ch. 11], as well as Abramowitz-Stegun [2, Ch. 8] for a list of useful formulas. The most results of this section are well-known and we give their brief proofs for the sake of completeness.

Let $P_{0}(x)=1, P_{1}(x)=x$. The Legendre polynomials $\left\{P_{n}(x)\right\}$ are defined by recurrence relations

$$
(n+1) P_{n+1}(x)=(2 n+1) x P_{n}(x)-n P_{n-1}(x), \quad n \geq 1 .
$$

It is important for us that

$$
\left(P_{n}, P_{m}\right)_{L^{2}[-1,1]}=\int_{-1}^{1} P_{n}(x) \overline{P_{m}(x)} d x=\frac{2}{2 n+1} \delta_{n m},
$$

where $\delta_{n m}$ is the Kronecker's delta. As usual, $(., .)_{L^{2}[-1,1]}$ stays for the scalar product in $L^{2}[-1,1]$. By Gram-Schmidt orthonormalization procedure applied to $\left\{x^{n}\right\}_{n \geq 0}$, the polynomials $\left\{p_{n}\right\}_{n \geq 0}$

$$
p_{n}(x)=\sqrt{\frac{2}{2 n+1}} P_{n}(x)
$$

form an orthonormal basis in $L^{2}[-1,1]$. To stick with more standard objects, we work with $\left\{P_{n}\right\}_{n \geq 0}$ keeping in mind the above renormalization condition.

We have for the derivatives $P_{n}^{\prime}(x)$, see [5, Sect. 10.10]

$$
\begin{aligned}
P_{n+1}^{\prime}(x) & =\sum_{k \geq 0, n-2 k \geq 0} \frac{2}{\left\|P_{n-2 k}\right\|^{2}} P_{n-2 k}(x) \\
& =\sum_{k \geq 0, n-2 k \geq 0}(2(n-2 k)+1) P_{n-2 k}(x) .
\end{aligned}
$$

It is important that the systems $\left\{x^{n}\right\}_{n \geq 0}$ and $\left\{P_{n}(x)\right\}_{n \geq 0}$ can be recalculated in terms of each other in a (relatively) simple manner. Let $E(x)$ be the entire (integer) part of $x \in \mathbb{R}$, and $m$ !! be the odd (or even) factorial, i.e.,

$$
(2 m+1) ! !=(2 m+1)(2 m-1) \ldots 1, \quad(2 m) ! !=(2 m)(2 m-2) \ldots 2 .
$$


Proposition 1.4 ([5, Sect. 10.10]). We have

$$
P_{n}(x)=\frac{1}{2^{n}} \sum_{k=0}^{E(n / 2)}(-1)^{k}\left(\begin{array}{l}
n \\
k
\end{array}\right)\left(\begin{array}{c}
2 n-2 k \\
n
\end{array}\right) x^{n-2 k},
$$

and

$$
P_{n}(x)=\frac{1}{2^{n}} \sum_{k=0}^{n}\left(\begin{array}{l}
n \\
k
\end{array}\right)^{2}(x-1)^{n-k}(x+1)^{k} .
$$

Proof. According to Rodrigues representation [5, Sect. 10.10],

$$
P_{n}(x)=\frac{1}{2^{n} n !}\left(\left(x^{2}-1\right)^{n}\right)^{(n)} .
$$

Applying the binomial formula to $\left(x^{2}-1\right)^{n}$ and taking the derivatives $n$ times, we come to

$$
\left(\left(x^{2}-1\right)^{n}\right)^{(n)}=\sum_{k=0}^{E(n / 2)}\left(\begin{array}{l}
n \\
k
\end{array}\right)(-1)^{k} \frac{(2 n-2 k) !}{(n-2 k) !} x^{n-2 k}
$$

Consequently,

$$
\begin{aligned}
P_{n}(x) & =\frac{1}{2^{n} n !} \sum_{k=0}^{E(n / 2)}(-1)^{k}\left(\begin{array}{l}
n \\
k
\end{array}\right) \frac{(2 n-2 k) !}{(n-2 k) !} x^{n-2 k} \\
& =\frac{1}{2^{n}} \sum_{k=0}^{E(n / 2)}(-1)^{k}\left(\begin{array}{l}
n \\
k
\end{array}\right)\left(\begin{array}{c}
2 n-2 k \\
n
\end{array}\right) x^{n-2 k}
\end{aligned}
$$

and so (1.6) is proved.

Writnig $\left(x^{2}-1\right)^{n}=(x-1)^{n}(x+1)^{n}$, and using once again the binomial formula and consecutive derivations, we obtain

$$
\begin{aligned}
\left(\left(x^{2}-1\right)^{n}\right)^{(n)} & =\sum_{k=0}^{n}\left(\begin{array}{l}
n \\
k
\end{array}\right)\left((x-1)^{k}\right)^{(k)}\left((x+1)^{n-k}\right)^{(n-k)} \\
& =\sum_{k=0}^{n}\left(\begin{array}{l}
n \\
k
\end{array}\right) \frac{n !}{(n-k) !}(x-1)^{n-k} \frac{n !}{k !}(x+1)^{k} .
\end{aligned}
$$

Hence,

$$
P_{n}(x)=\frac{1}{2^{n}} \sum_{k=0}^{n}\left(\begin{array}{l}
n \\
k
\end{array}\right)^{2}(x-1)^{n-k}(x+1)^{k},
$$

and (1.7) is proved as well.

It is rather remarkable that these formulas can be explicitly inverted. Despite its neat form, we were unable to find this result in the literature, and for this reason we prefer to give its proof.

Proposition 1.5 ([17]). For $n \geq 1$, we have

$$
x^{n}=\sum_{k=n, n-2, \ldots} \frac{(2 k+1) n !}{2^{(n-k) / 2}((n-k) / 2) !(k+n+1) ! !} P_{k}(x) .
$$

Proof. Relation (1.8) yields obviously $P_{0}(x)=1$ and $P_{1}(x)=x$. To argue by recurrence, we suppose that

$$
x^{n}=\sum_{k=n, n-2, \ldots} \frac{(2 k+1) n !}{2^{(n-k) / 2}((n-k) / 2) !(k+n+1) ! !} P_{k}(x),
$$


and we want to show

$$
x^{n+1}=\sum_{k=n+1, n-1, \ldots} \frac{(2 k+1)(n+1) !}{2^{(n+1-k) / 2}((n+1-k) / 2) !(k+n+2) ! !} P_{k}(x) .
$$

Reminding (1.3), we see

$$
x P_{k}(x)=\frac{k}{2 k+1} P_{k-1}(x)+\frac{k+1}{2 k+1} P_{k+1}(x) .
$$

Writing $x^{n+1}=x\left(x^{n}\right)$ and plugging the above relation in (1.9), we obtain

$$
\begin{aligned}
x^{n+1}= & \sum_{k=n, n-2, \ldots} \frac{(2 k+1) n !}{2^{(n-k) / 2}((n-k) / 2) !(k+n+1) ! !} x P_{k}(x) \\
= & \sum_{k=n, n-2, \ldots} \frac{(2 k+1) n !}{2^{(n-k) / 2}((n-k) / 2) !(k+n+1) ! !}\left(\frac{k+1}{2 k+1} P_{k+1}(x)\right. \\
& \left.+\frac{k}{2 k+1} P_{k-1}(x)\right) \\
= & \sum_{k=n+1, n-1, \ldots} \frac{k n !(k+n+2)}{2^{(n+1-k) / 2}((n+1-k) / 2) !(k+n+2) ! !} P_{k}(x) \\
& +\sum_{k=n-1, n-3, \ldots} \frac{(k+1) n !(n+1-k)}{2^{(n-1-k) / 2}((n+1-k) / 2) !(k+n+2) ! !} P_{k}(x) \\
= & \frac{(n+1) n !(2 n+3)}{(2 n+3) ! !} P_{n+1} \quad(2 k+1)(n+1) ! \\
& +\sum_{k=n-1, n-3, \ldots} \frac{2^{(n-1-k) / 2}((n+1-k) / 2) !(k+n+2) ! !}{} P_{k}(x) .
\end{aligned}
$$

after a series of elementary transformations. The proposition is proved.

For the sequel, it will be convenient to specify the formula of the above proposition to the case on even and odd $n$. That is, for $n$ even, $n=2 m$, we have

$$
\begin{aligned}
x^{2 m} & =\sum_{k=0}^{m} \frac{(4 k+1)(2 m) !}{2^{m-k}(m-k) !(2 k+2 m+1) ! !} P_{2 k}(x) \\
& =\sum_{k=0}^{m} \frac{(4 k+1)(2 m) !}{(2 m-2 k) ! !(2 k+2 m+1) ! !} P_{2 k}(x) \\
& =\sum_{k=0}^{m} b_{2 m, 2 k} P_{2 k}(x),
\end{aligned}
$$

where

$$
b_{2 m, 2 k}=\frac{(4 k+1)(2 m) !}{(2 m-2 k) ! !(2 k+2 m+1) ! !}, \quad k=0, \ldots, m .
$$

Similarly, for $n$ odd, $n=2 m+1$,

$$
\begin{aligned}
x^{2 m+1} & =\sum_{k=0}^{m} \frac{(4 k+3)(2 m+1) !}{2^{m-k}(m-k) !(2 k+2 m+3) ! !} P_{2 k+1}(x) \\
& =\sum_{k=0}^{m} \frac{(4 k+3)(2 m+1) !}{(2 m-2 k) ! !(2 k+2 m+3) ! !} P_{2 k+1}(x) \\
& =\sum_{k=0}^{m} b_{2 m+1,2 k+1} P_{2 k+1}(x),
\end{aligned}
$$


where

$$
b_{2 m+1,2 k+1}=\frac{(4 k+3)(2 m+1) !}{(2 m-2 k) ! !(2 k+2 m+3) ! !}, \quad k=0, \ldots, m .
$$

2. Some Sufficient Conditions for RAdial Toeplitz to LiE IN Schatten-von Neumann Classes $\mathcal{S}_{p}, 0<p<\infty$

Let the function $\varphi$ be defined on $I=[0,1]$. Theorem 1.3 suggests is that if the symbol $\varphi$ is continuous in a (left) neighborhood of point $x=1$ and $\varphi(1)=0$, then obviously $\lim _{n \rightarrow+\infty} c_{n}(\varphi)=0$.

Suppose now that $\varphi$ is $l$ times continuously differentiable on $I, l \in \mathbb{N}$. One can easily see that the conditions $\varphi^{(k)}(1)=0, k=0, \ldots, l-1$, imply a faster decay of $c_{n}(\varphi)$ when $n \rightarrow+\infty$.

To formulate the coming proposition, denote by $W^{l, p}(I)$ the Sobolev space on the interval $I$, i.e., $f \in W^{l, p}(I)$ iff

$$
\|f\|_{W^{l, p}}=\sum_{j=0}^{l}\left\|f^{(j)}\right\|_{p}<\infty
$$

where the derivatives $f^{(j)}$ are understood in the distributional sense, and the norms $\|.\|_{p}$ refer to $L^{p}(I), p \geq 1$. See Adams [1] for more information on the subject.

The proposition is completely elementary, and we give it for the sake of completeness only.

Proposition 2.1. Let $l \in \mathbb{N}, p \geq 1$. Let $\varphi \in W^{l, p}(I)$ and $\varphi^{(k)}(1)=0, k=$ $0, \ldots, l-1$. Then

where $C=C(l, p)$.

$$
\left|c_{n}(\varphi)\right| \leq \frac{C}{n^{l-1 / p}}\|\varphi\|_{W^{l, p}}
$$

Proof. As usual, for $p \geq 1$, we set $q=p /(p-1), q \geq 1$. Starting with $l=1$, we see that

$$
\begin{aligned}
c_{n}(\varphi) & =2(n+1) \int_{0}^{1} \varphi(x) x^{2 n+1} d x=\int_{0}^{1} \varphi(x) d\left(x^{2(n+1)}\right) \\
& =-\int_{0}^{1} \varphi^{\prime}(x) x^{2(n+1)} d x .
\end{aligned}
$$

By Hölder inequality

$$
\left|c_{n}(\varphi)\right| \leq\left\|\varphi^{\prime}\right\|_{p}\left\|x^{2(n+1)}\right\|_{q} \leq C\|\varphi\|_{W^{1, p}} \cdot \frac{1}{n^{1 / q}}
$$

where $C=C(l, p)$. For $l=1$, one has $1 / q=1-1 / p=l-1 / p$.

For general $l$, the integration by parts applied $(l-1)$ times to the above integral gives

$$
c_{n}(\varphi)=\frac{(-1)^{l}}{(2 n+3)(2 n+4) \ldots(2 n+1+l)} \int_{0}^{1} \varphi^{(l)}(x) x^{2 n+1+l} d x .
$$

Consequently, we get by Hölder inequality

$$
\left|c_{n}(\varphi)\right| \leq \frac{C}{n^{l-1+1 / q}}\left\|\varphi^{(l)}\right\|_{p} \leq C\|\varphi\|_{W^{l, p}} \frac{1}{n^{l-1 / p}}
$$

where $C=C(l, p)$.

Suppose that $l-1 / p>0$. It follows immediately that $T_{\varphi} \in \mathcal{S}_{\alpha}, \alpha>1 /(l-1 / p)$, for symbols $\varphi$ from the above proposition, and, moreover

$$
\left\|T_{\varphi}\right\|_{\mathcal{S}_{\alpha}} \leq C\|\varphi\|_{W^{l, p}}
$$


Let us observe also that Proposition 2.1 is a sufficient condition for the decay of $\left\{c_{n}(\varphi)\right\}$, but it is not at all a necessary one. For instance, it explains how the coefficients $\left\{c_{n}(\varphi)\right\}$ can decay when $\varphi \geq 0$ while no cancellations in integral (1.2) defining $c_{n}(\varphi)$ are possible. When $\varphi$ is complex (or real and sign-indefinite), the integral giving $c_{n}(\varphi)$ can become small because of very fast oscillations of $\varphi$ in a (left) neighborhood of $x=1$, see Proposition 2.3.

The lemma is a simple calculation based on well-known properties $\Gamma$-function.

Lemma 2.2. Let $\delta>-1$. Then

$$
I_{\delta}(n)=\int_{0}^{1} s^{n}(1-s)^{\delta} d s \leq \frac{C \Gamma(\delta+1)}{n^{\delta+1}},
$$

where $C$ is an absolute constant.

Proof. We give a brief sketch of the proof for the completeness. Take $a_{n}=$ $1 / \sqrt{n+1}$. Making the change of variable $s=e^{-t}$ in $I_{\delta}(n)$ and writing the integral on $\left[0, a_{n}\right)$ and $\left[a_{n},+\infty\right)$, we get

$$
\begin{aligned}
\int_{0}^{1} s^{n}(1-s)^{\delta} d s & =\int_{0}^{\infty} e^{-n t}\left(1-e^{-t}\right)^{\delta} d t=I_{1}+I_{2} \\
& :=\int_{0}^{a_{n}} e^{-n t}\left(1-e^{-t}\right)^{\delta} d t+\int_{a_{n}}^{\infty} e^{-n t}\left(1-e^{-t}\right)^{\delta} d t
\end{aligned}
$$

One sees easily that

$$
I_{2} \leq \frac{C}{\sqrt{n+1}} e^{-(n+1) a_{n}}
$$

For any $\epsilon>0$ and $t$ small enough, we have $\left(1-e^{-t}\right) \leq(1+\epsilon) t$, and so

$$
\begin{aligned}
I_{1} & \leq(1+\epsilon)^{\delta} \int_{0}^{a_{n}} e^{-n t} t^{\delta} d t=\frac{(1+\epsilon)^{\delta}}{(n+1)^{\delta}} \int_{0}^{(n+1) a_{n}} e^{-u} u^{\delta} d u \\
& \rightarrow \frac{(1+\epsilon)^{\delta} \Gamma(1+\delta)}{(n+1)^{\delta}}
\end{aligned}
$$

where $u=(n+1) t$ and $n \rightarrow+\infty$. The lemma is proved.

Of course, one can prove with a little more effort that $I_{\delta}(n) \asymp \Gamma(\delta+1) / n^{\delta+1}$ for $n \rightarrow+\infty$.

The first two points of the following proposition are borrowed from GrudskyVasilevski [8, Theorem 3.3]. The third point addresses the fact whether the Toeplitz operator $T_{\varphi}$ lies in $\mathcal{S}_{p}, 0<p<\infty$, and it is obtained with the help of techniques similar to those of [8]. Notice that neither the continuity, nor even the boundedness of the symbol $\varphi$ in the neighborhood of $x=1$ are required. We also warn the reader that our notation differs from that one of the latter paper.

We set $\Phi(x)=\int_{x}^{1} \varphi(s) d s$.

Proposition 2.3. Assume that $\varphi \in L^{1}[0,1]$. We have the following implications (one understands that $s \rightarrow 1-0$ ):

(1) If $|\Phi(s)| \leq C(1-s)$, then $\sup _{n}\left|c_{n}(\varphi)\right|<\infty$ (i.e., the operator $T_{\varphi}$ is bounded).

(2) If

$$
\lim _{s \rightarrow 1-0} \frac{|\Phi(s)|}{(1-s)}=0,
$$

then $\lim _{n \rightarrow+\infty}\left|c_{n}(\varphi)\right|=0$ (i.e., the operator $T_{\varphi}$ is compact). 
(3) Let

$$
|\Phi(s)| \leq C(1-s)^{\delta}
$$

for $a \delta>1$. Then

$$
\left|c_{n}(\varphi)\right| \leq \frac{C}{n^{(\delta-1)}},
$$

i.e., the operator $T_{\varphi}$ is in the Schatten-von Neumann class $\mathcal{S}_{p+\epsilon}, p=1 /(\delta-$ 1) for any $\epsilon>0$.

Proof. For (1), (2), see Grudsky-Vasilevski [8, Theorem 3.3]. Turning to (3), we make integration by parts in (1.2)

$$
c_{n}(\varphi)=(2 n+2)(2 n+1) \int_{0}^{1} \Phi(x) x^{2 n} d x .
$$

By hypothesis of (3), there is a $\delta_{0}>0$ such that $|\Phi(x)| \leq C(1-x)^{\delta}$ for $1-\delta_{0} \leq x<1$. We re-write the latter integral as

$$
\begin{aligned}
c_{n}(\varphi) & =(2 n+2)(2 n+1) \int_{0}^{1} \Phi(x) x^{2 n} d x \\
& =(2 n+2)(2 n+1)\left(\int_{0}^{1-\delta_{0}} \Phi(x) x^{2 n} d x+\int_{1-\delta_{0}}^{1} \Phi(x) x^{2 n} d x\right) \\
& =: I_{1}+I_{2},
\end{aligned}
$$

the factor $(2 n+2)(2 n+1)$ being absorbed in the integrals. Since $\varphi \in L^{1}[0,1], \Phi$ is bounded and so $I_{1}$ decays with exponential rate, i.e.,

$$
I_{1} \leq C(2 n+2)(2 n+1)\left(1-\delta_{0}\right)^{2 n} \text {. }
$$

Turning to $I_{2}$, we see

$$
\begin{aligned}
I_{2} & \leq C(2 n+2)(2 n+1) \int_{1-\delta_{0}}^{1}(1-x)^{\delta} x^{2 n} d x \\
& \leq C(2 n+2)(2 n+1) \int_{0}^{1}(1-x)^{\delta} x^{2 n} d x \leq \frac{C \Gamma(\delta+1) n^{2}}{n^{\delta+1}} \leq \frac{C}{n^{\delta-1}},
\end{aligned}
$$

where at the last step we used the above lemma.

Now, we consider

$$
\varphi(x)=\varphi_{\alpha, \beta}(x)=(1-x)^{-\beta} \sin (1-x)^{-\alpha},
$$

where $\alpha>0$ and $\beta<1$. Of course, the condition on $\beta$ guarantees that $\varphi \in L^{1}[0,1]$. The computations from [8, p. 23] (see equation (3.3) and relations below) give that

$$
\Phi(x)=\frac{1}{\alpha}(1-x)^{\alpha-\beta+1} \cos (1-x)^{-\alpha}+O\left((1-x)^{2 \alpha-\beta+1}\right),
$$

so condition (2.1) is satisfied with $\delta=\alpha-\beta+1>0$, and, consequently, point (3) of Proposition 2.3 holds. Notice that we may pick $\delta$ as large as we want taking $\alpha>0$ big enough.

Corollary 2.4. There is a symbol $\varphi=\varphi_{\alpha, \beta}(2.2)$ with obvious choice of parameters $\alpha, \beta$ having the properties:

(1) $\varphi \in L^{1}[0,1]$

(2) the operator $T_{\varphi}$ is compact,

(3) for any $\delta_{0}>0, \sup _{\left[1-\delta_{0}, 1\right)}|\varphi(x)|=+\infty$,

(4) moreover, for any $p>0$, there are $\alpha>0$ and $\beta<1$, such that $T_{\varphi_{\alpha, \beta}} \in \mathcal{S}_{p}$. 


\section{The solution to the spectral problem for Radial Toeplitz OPERATORS AND LEGENDRE POLYNOMIALS}

3.1. Direct and inverse spectral problems for radial Toeplitz operators. Recall that for a given radial function $\varphi \in L^{1}([0,1], x d x)$, the Toeplitz operator (0.1) has a diagonal matrix with coefficients $\left\{c_{n}(\varphi)\right\}(1.2)$ in the standard basis of $L_{a}^{2}(\mathbb{D})$. So the spectrum $\sigma\left(T_{\varphi}\right)$ coincides with $\overline{\left\{c_{n}(\varphi)\right\}}$.

The spectral problem for these operators consists of two parts: the first part (i.e., the direct spectral problem) is to understand the properties of $\sigma\left(T_{\varphi}\right)$ (or to compute the spectrum in a rather explicit manner) for a given $\varphi$. The second part (i.e., the inverse direct problem) is to reconstruct the symbol $\varphi$ (if possible), from the spectrum $\sigma\left(T_{\varphi}\right)$ of the Toeplitz operator. As we show, this problem admits a neat solution in terms of Legendre polynomials $\left\{P_{n}(x)\right\}$ in $L^{2}[-1,1]$.

Before giving the construction, we make some reductions. First, recalling formula (1.2), we make the change of variable $x=\sqrt{s}$ and we set $\psi(s)=\phi(\sqrt{s})$, so

$$
C_{n}(\psi):=c_{n}(\varphi)=2(n+1) \int_{0}^{1} \varphi(x) x^{2 n+1} d x=(n+1) \int_{0}^{1} \psi(s) s^{n} d s .
$$

We accept the notation from the above relation for the rest of the paper.

Second, we wish to represent the function $\psi$ as a series of Legendre polynomials on $[-1,1]$. The rescaling the polynomials $\left\{P_{n}\right\}$ to the interval $[-1,1]$ leads to some cumbersome relations; to keep the formulas we work with reasonably simple, we prefer to extend the function $\psi$ to $[-1,1]$ in appropriate ways.

Namely, for a $\psi \in L^{p}[0,1], p \geq 1$, we set

$$
\psi_{e}(s)=\left\{\begin{array}{ll}
\psi(x), & x \geq 0, \\
\psi(-x), & x<0,
\end{array} \quad \psi_{o}(s)= \begin{cases}\psi(x), & x \geq 0, \\
-\psi(-x), & x<0\end{cases}\right.
$$

Of course, we have $\psi=\psi_{e}+\psi_{o}$ on $[-1,1]$, where $\psi$ is extended by zero to $[-1,0)$, and $\left\|\psi_{e}\right\|_{L^{p}[-1,1]}^{p}=\left\|\psi_{o}\right\|_{L^{p}[-1,1]}^{p}=2\|\psi\|_{L^{p}[0,1]}^{p}$. It follows

$$
C_{n}(\psi)=(n+1) \int_{0}^{1} \psi(s) s^{n} d s=\frac{(n+1)}{2} \int_{-1}^{1} \psi_{e}(s) s^{n} d s=: C_{n}\left(\psi_{e}\right)
$$

for $n$ even and $C_{n}\left(\psi_{e}\right)=0$ for $n$ odd. Similarly,

$$
C_{n}(\psi)=(n+1) \int_{0}^{1} \psi(s) s^{n} d s=\frac{(n+1)}{2} \int_{-1}^{1} \psi_{o}(s) s^{n} d s=: C_{n}\left(\psi_{o}\right)
$$

for $n$ odd and $C_{n}\left(\psi_{o}\right)=0$ for $n$ even. In particular, $C_{n}(\psi)=C_{n}\left(\psi_{e}\right)$ for $n$ even, and $C_{n}(\psi)=C_{n}\left(\psi_{0}\right)$ for $n$ odd.

Assume now $\psi(s)=\sum_{k=0}^{N} a_{k} P_{k}(s)$ on $[-1,1]$. Then, we get for even $m=2 n$

$$
\begin{aligned}
C_{2 n}(\psi) & =\frac{(2 n+1)}{2} \int_{-1}^{1} \psi_{e}(x) x^{2 n} d x \\
& =\frac{(2 n+1)}{2} \int_{-1}^{1}\left(\sum_{j=0}^{N} a_{j} P_{j}(x)\right)\left(\sum_{k=0}^{n} b_{2 n, 2 k} P_{2 k}(x)\right) d x \\
& =\frac{(2 n+1)}{2} \int_{-1}^{1} \sum_{j=0}^{[N / 2]} \sum_{k=0}^{n} b_{2 n, 2 k} a_{2 j}=P_{2 j}(x) P_{2 k}(x) d x \\
& =\sum_{k=0}^{\min \{n,[N / 2]\}}\left(\frac{2 n+1}{4 k+1} b_{2 n, 2 k}\right) a_{2 k}=: \sum_{k=0}^{\min \{n,[N / 2]\}} d_{2 n, 2 k} a_{2 k},
\end{aligned}
$$


where

$$
d_{2 n, 2 k}=\frac{(2 n+1) !}{(2 n-2 k) ! !(2 n+2 k+1) ! !} .
$$

For odd $m=2 n+1$, we obtain

$$
\begin{aligned}
C_{2 n+1}(\psi) & =\frac{(2 n+2)}{2} \int_{-1}^{1} \psi_{o}(x) x^{2 n+1} d x \\
& =\frac{(2 n+2)}{2} \int_{-1}^{1}\left(\sum_{j=0}^{N} a_{j} P_{j}(x)\right)\left(\sum_{k=0}^{n} b_{2 n+1,2 k+1} P_{2 k+1}(x)\right) d x \\
& =\frac{(2 n+2)}{2} \int_{-1}^{1} \sum_{j=0}^{[N / 2]} \sum_{k=0}^{n} b_{2 n+1,2 k+1} a_{2 j+1} P_{2 j+1}(x) P_{2 k+1}(x) d x \\
& =\sum_{k=0}^{\min \{n,[N / 2]\}}\left(\frac{2 n+2}{4 k+3} b_{2 n+1,2 k+1}\right) a_{2 k+1}=: \sum_{k=0}^{\min \{n,[N / 2]\}} d_{2 n+1,2 k+1} a_{2 k+1},
\end{aligned}
$$

where

$$
d_{2 n+1,2 k+1}=\frac{(2 n+2) !}{(2 n-2 k) ! !(2 n+2 k+3) ! !} .
$$

In the sequel, we will be concerned with bounds on the coefficients $C_{n}(\psi)$ for different functions $\psi$. Observe that the coefficients $C_{2 n}(\psi)(3.2)$ depend on $d_{2 n, 2 k}$ and $a_{2 k}$ only, and the same applies to $C_{2 n+1}(\psi)(3.4)$ and $d_{2 n+1,2 k+1}, a_{2 k+1}$, respectively. Hence, we have a nice separation of variables. Since the computations of $\left\{C_{n}(\psi)\right\}$ for $n$ even or odd are similar, we concentrate on the the case of even $n$. The case of odd $n$ goes through likewisely and is omitted.

Let $\psi(s)=\sum_{k=0}^{N} a_{k} P_{k}(s), s \in[-1,1]$. Consider the map given by

$$
\left\{c_{n}(\varphi)\right\}=\left\{C_{2 n}(\psi) ; C_{2 n+1}(\psi)\right\}=\mathcal{D}\left(\left\{a_{k}\right\}\right),
$$

where $\mathcal{D}($.$) is the multiplication by \mathcal{D}=\left[d_{n, k}\right]_{n, k}$, see (3.2) and (3.4) for the definition of $d_{n, k}$. We call the map the direct spectral map for the operator $T_{\varphi}$; to keep the notation simple, we denote the map and its matrix (in the fixed basis $\left\{P_{n}\right\}$ ) by the same letter $\mathcal{D}$. The inverse spectral map for the operator $T_{\varphi}$ is

$$
\left\{a_{k}\right\}=\mathcal{I}\left(\left\{c_{n}(\varphi)\right\}\right)=\mathcal{I}\left(\left\{C_{2 n}(\psi) ; C_{2 n+1}(\psi)\right\}\right),
$$

and it can be readily written down by means of formulas (1.6), (1.7). One of the main points of the present section is to study the direct spectral map $\mathcal{D}$; the properties of the inverse spectral map $\mathcal{I}$ can be treated from a similar point of view, but we do not pursue in this direction here.

We assume that $\psi \in L^{2}[-1,1]$, or, equivalently,

$$
\|\psi\|_{L^{2}[-1,1]}^{2}=\sum_{k=0}^{\infty} \frac{2}{2 k+1}\left|a_{k}\right|^{2}<+\infty .
$$

The corresponding weighted $l^{2}$-space is denoted by $l^{2}(\{2 /(2 k+1)\})$. It follows immediately from the definition of $\mathcal{S}_{p}, p>0$, that $T_{\varphi} \in \mathcal{S}_{p}$ iff $\left\{c_{n}(\varphi)\right\} \in l^{p}, p>0$.

We wish to understand what sequences $\left\{c_{n}(\varphi)\right\}_{n}$ can be generated by functions $\psi \in L^{2}[-1,1]$ with the help of map $\mathcal{D}$, see $(3.5)$. 
3.2. Some auxiliary results and the proof of Theorem 3.3. As explained above, we do the computations for the sequences with even indices, i.e., we suppose $\psi=\sum_{k=0}^{\infty} a_{2 k} P_{2 k}$. We denote by $\mathcal{D}_{e}$ the restriction of the map $\mathcal{D}$ to the evenindexed subsequences, i.e.,

$$
\left\{C_{2 n}(\psi)\right\}_{n}=\mathcal{D}_{e}\left(\left\{a_{2 k}\right\}_{k}\right) .
$$

Notice also that, for any $n$,

$$
d_{2 n, 0}=1, \quad d_{2 n, 2 n}=\frac{(2 n+1) !}{(4 n+1) ! !} \rightarrow 0(n \rightarrow \infty),
$$

and

$$
d_{2 n, 2 k+2}=d_{2 n, 2 k} \frac{2 n-2 k}{2 n+2 k+3} .
$$

Lemma 3.1. We have:

(1) the matrix of $\mathcal{D}_{e}$ is lower-triangular, i.e., $d_{2 n, 2 k}=0, k>n$, and $d_{2 n, 2 k}>0$ for $0 \leq k \leq n$. Moreover:

(a) for any fixed $n, d_{2 n, 2 k}$ is decaying with respect to $k$,

(b) for any fixed $k, \lim _{n \rightarrow+\infty} b_{2 n, 2 k}=1$.

(2) We have

$$
d_{2 n, 2 k} \leq \exp \left(-C k^{2} / n\right)
$$

with an absolute constant $C>0$.

Proof. Claim (1) of the lemma is clear from (3.3). Claim (2) is also easy to prove and it follows from (3.7). Indeed, we have

$$
\begin{aligned}
d_{2 n, 2 k} & =\prod_{j=0}^{k-1}\left(\frac{2 n-2 j}{2 n+2 j+3}\right)=\exp \left(\sum_{j=0}^{k-1} \log \left(\frac{2 n-2 j}{2 n+2 j+3}\right)\right) \\
& \leq \exp \left(-\sum_{j=0}^{k-1} \frac{4 j+3}{2 n+2 j+3}\right) \leq \exp \left(-\frac{1}{2 n+2 k+1} \sum_{j=0}^{k-1}(4 j+3)\right) \\
& \leq \exp \left(-C \frac{k^{2}}{n}\right)
\end{aligned}
$$

where we used that $0 \leq j \leq k \leq n$.

The form of the map $\mathcal{D}_{e}$ suggests that it might have very nice cancellation properties, that is, for an appropriate choice a sequence $\left\{a_{2 k}\right\} \in l^{2}(\{2 /(2 k+1)\})$, the corresponding sequence $\left\{C_{2 n}(\psi)\right\}=\mathcal{D}_{e}\left(\left\{a_{2 k}\right\}\right)$ could be in $l^{p}, 0<p<\infty$.

Lemma 3.2. Fix a $p \in \mathbb{N}$. There is a set coefficients $\left(A_{j}(.)\right)_{j=0, \ldots, p}, A_{j}()=.A_{p, j}($. considered as functions of $k$, with the following properties:

(1) For all $n, k$, we have

$$
\sum_{j=0}^{p} d_{2 n, 2(k+j)} A_{j}(k)=d_{2 n, 2 k} \prod_{j=1}^{p} \frac{1}{(2 n+2(k+j)+1)} .
$$

(2) $A_{j}(k)=S_{j}(k) / T_{p}(k)$, where $S_{j}=S_{j, p}, T_{p}$ are polynomials with respect to $k, j=0, \ldots, p, \operatorname{deg} T_{p}=p(p+1) / 2$ and

$$
\operatorname{deg} S_{0}=\operatorname{deg} S_{1}=p(p+1) / 2-1, \quad \operatorname{deg} S_{j}=p(p+1) / 2-j,
$$

where $j=2, \ldots, p$. In particular, the rational functions $\left\{A_{j}(.)\right\}_{j=0, \ldots, p}$ have the degrees $\operatorname{deg} A_{0}=\operatorname{deg} A_{1}=-1, \operatorname{deg} A_{j}=-j, j=2, \ldots, p$.

(3) The zeroes of polynomials $\left\{S_{j}\right\}_{j=0, \ldots, p}, T_{p}$ depend on $p$ only; in particular, they do not depend neither on $k$, nor on $n$. 
(4) It follows that, for a fixed $n$, and $k$ large enough

$$
\begin{aligned}
& \quad C_{1} \leq k\left|A_{0}(k)\right| \leq C_{2}, C_{1} \leq k\left|A_{1}(k)\right| \leq C_{2}, C_{1} \leq k^{j}\left|A_{j}(k)\right| \leq C_{2}, \\
& \text { where } j=2, \ldots, p \text { and } C_{1}, C_{2}>0 .
\end{aligned}
$$

Proof. To illustrate the conclusion of the lemma, let us take $p=1$ first. So, we want to pick $A_{0}(k)$ and $A_{1}(k)$ with the property

$$
d_{2 n, 2 k} A_{0}(k)+d_{2 n, 2(k+1)} A_{1}(k)=d_{2 n, 2 k} \frac{1}{2 n+2 k+3} .
$$

Rewriting the LHS of this relation as

$$
\cdots=d_{2 n, 2 k}\left(A_{0}(k)+A_{1}(k) \frac{(2 n-2 k)}{(2 n+2 k+3)}\right),
$$

we see that

$$
A_{0}(k)=\frac{1}{4 k+3}, A_{1}(k)=-\frac{1}{4 k+3},
$$

and these $A_{0}, A_{1}$ satisfy the claim of the lemma.

The computation for a general $p \in \mathbb{N}$ is rather lengthy and tedious, though completely elementary. We give a sketch of the proof in this situation leaving the technical details to the reader.

Pick an arbitrary $p \in \mathbb{N}, p>1$. At the first stage of the construction, we seek for coefficients $\left\{B_{j}\right\}_{j=1, \ldots, p}$, depending on $p$ only, such that

$$
F_{p}(n):=F_{p, k}(n)=\frac{1}{\prod_{j=1}^{p}(2 n+2(k+j)+1)}=\sum_{j=1}^{p} \frac{B_{j}}{(2 n+2(k+j)+1)}
$$

We consider the function $F_{p}$ as a (rational) function of $n$; $k$ and $p$ are fixed parameters. Equating the residues at the poles on the RHS and on the LHS of (3.10), we express the coefficients $B_{j}$ as

$$
B_{j}=\operatorname{Res}_{(-2(k+j)-1) / 2} F_{p}(n)=\frac{(-1)^{j-1}}{2^{j-1}(j-1) !} \cdot \frac{1}{2^{p-j}(p-j) !},
$$

where $j=1, \ldots, p$.

Going to the second stage of the computation, we seek the coefficients $\left\{A_{j}(.)\right\}_{j=1, \ldots, p}$ with the property

$$
\begin{aligned}
\sum_{j=0}^{p} d_{2 n, 2(k+j)} A_{j}(k) & =d_{2 n, 2 k} \frac{1}{\prod_{j=1}^{p}(2 n+2(k+j)+1)} \\
& =d_{2 n, 2 k} \sum_{s=1}^{p} \frac{B_{s}}{(2 n+2(k+s)+1)} .
\end{aligned}
$$

We define the function $G_{p}(n):=G_{p, k}(n)$ as

$$
G_{p}(n)=\frac{1}{d_{2 n, 2 k}} \sum_{j=0}^{p} d_{2 n, 2(k+j)} A_{j}(k) .
$$

Relation (3.11) reads as

$$
G_{p}(n)=\sum_{j=0}^{p} A_{j}(k) \prod_{s=1}^{j}\left(\frac{2 n-2(k+s-1)}{2 n+2(k+s)+1}\right) .
$$

Of course,

$$
\frac{2 n-2(k+s-1)}{2 n+2(k+s)+1}=1-\frac{4(k+s)-1}{2 n+2(k+s)+1}
$$


and hence

$$
\text { LHS of }(3.11)=\sum_{j=0}^{p} A_{j}\left\{\prod_{s=1}^{j}\left(1-\frac{4(k+s)-1}{(2 n+2(k+s)+1)}\right)\right\} .
$$

As at the first stage of the proof of the lemma, we equate the residues on the RHS and on the LHS of (3.13). That is, we have

$$
\begin{aligned}
\operatorname{Res}_{-(2(k+j)+1) / 2} G_{p}(n) & =A_{j}\left\{(-1)^{j}\left(\frac{4(k+j)-1}{2}\right) \prod_{s=1, s \neq j}^{j}\left(\frac{4 k+2(s+j)-1}{2(s-j)}\right)\right\} \\
+ & \ldots+A_{r}\left\{(-1)^{r}\left(\frac{4(k+j)-1}{2}\right) \prod_{s=1, s \neq j}^{r}\left(\frac{4 k+2(s+j)-1}{2(s-j)}\right)\right\} \\
+ & \ldots+A_{p}\left\{(-1)^{p}\left(\frac{4(k+j)-1}{2}\right) \prod_{s=1, s \neq j}^{p}\left(\frac{4 k+2(s+j)-1}{2(s-j)}\right)\right\} \\
& =\frac{B_{j}}{2},
\end{aligned}
$$

where $j=1, \ldots, p$, and $j \leq r \leq p$.

Now, it is convenient to set

$$
\mathcal{A}=\left[A_{1}, A_{2}, \ldots, A_{p}\right]^{t}, \quad \mathcal{B}=\left[B_{1}, \ldots, B_{p}\right]^{t},
$$

and $p \times p$ matrix $\mathcal{C}$ with entries

$$
\mathcal{C}_{j, r}= \begin{cases}(-1)^{r}(4(k+j)-1) \prod_{s=0, s \neq j}^{r}\left(\frac{4 k+2(s+j)-1}{2(s-j)}\right), & t \geq j, \\ 0 & , \quad r<j,\end{cases}
$$

Notice that the matrix $\mathcal{C}$ is upper triangular; in particular, we have

$$
T_{p}(k):=\operatorname{det} \mathcal{C}=\prod_{j=1}^{p} \mathcal{C}_{j j} \neq 0
$$

for $k$ large enough. As relation (3.14) clearly indicates, every entry $\mathcal{C}_{j j}=\mathcal{C}_{j j}(k)$ is a polynomial with respect to $k$ of degree $j, j=1, \ldots, p$. So, $\operatorname{deg} T_{p}=p(p+1) / 2$, as claimed in the lemma.

The system of equations on $\left\{A_{j}\right\}_{j=1, \ldots, p}$, see $(3.11)$, reads as $\mathcal{C} \cdot \mathcal{A}=\mathcal{B}$ and one has to add equation $A_{0}=-\sum_{j=1}^{p} A_{j}$ defining $A_{0}$ to it. Trivially, we solve this system with the help of Cramer's rule, i.e.,

$$
A_{j}(k)=\frac{\operatorname{det} \mathcal{C}_{j, \mathcal{B}}}{\operatorname{det} \mathcal{C}}=: \frac{S_{j, p}(k)}{T_{p}(k)},
$$

where $S_{j}(k)=S_{j, p}(k)=\operatorname{det} \mathcal{C}_{j, \mathcal{B}}$ is a polynomial of $k$, and $\mathcal{C}_{j, \mathcal{B}}$ is the $p \times p$ matrix obtained from $\mathcal{C}$ with its $j$-th column replaced by $\mathcal{B}$. It is easy to see that $\operatorname{deg} S_{j}=p(p+1) / 2-j$.

Claim (3) of the lemma follows at once from (1) and (2).

Theorem 3.3. Take an arbitrary $\delta_{0}>0$. There exists a $\varphi \in L^{2}([0,1], x d x)$ such that

(1) $\varphi^{\prime} \notin L^{2}[0,1]$,

(2) the Toeplitz operator $T_{\varphi}(0.1)$ with radial symbol $\varphi$ lies in $\mathcal{S}_{\delta_{0}}$.

Proof. For a given $\delta_{0}>0$, pick a $p \in \mathbb{N}$ so that $1 /(p-1)<\delta_{0}$. Take $N_{0}$ large enough to guarantee that the coefficients $\left(A_{j}(k)\right)_{j=0, \ldots, p}$ are well-defined for $k \geq(p+1) N_{0}$, see Lemma 3.2, and especially its claims (2) and (3). 
We define the sequence $\left\{a_{2 k}\right\}_{k}$ using the coefficients $\left\{A_{j}(k)\right\}_{j=0, \ldots, p}$ from the above lemma. We have $k=(p+1) s+r$, where $0 \leq r<p+1$, and we set

$$
a_{2 k}:=A_{r}((p+1) s), \quad k \geq(p+1) N_{0} .
$$

or, equivalently

$$
\begin{aligned}
& \left(a_{2(p+1) s}, a_{2((p+1) s+1)}, \ldots a_{2((p+1) s+p)}\right) \\
& := \begin{cases}(0, \ldots, 0) & , \quad s<N_{0}, \\
\left(A_{0}((p+1) s), A_{1}((p+1) s), \ldots A_{p}((p+1) s)\right. & , \quad s \geq N_{0} .\end{cases}
\end{aligned}
$$

We set now

$$
\psi_{e}(x)=\sum_{n=0}^{\infty} a_{2 n} P_{2 n}(x),
$$

recall that this $\psi$ defines the function $\varphi$ on $[0,1]$ by means of $(3.1)$. We claim that $\psi$ (and hence $\varphi$ ) possesses the properties stated in the theorem. Indeed,

$$
\begin{aligned}
\|\psi\|_{L^{2}[0,1]}^{2} & =\frac{1}{2}\left\|\psi_{e}\right\|_{L^{2}[-1,1]}^{2}=\frac{1}{2} \sum_{n}\left|a_{2 n}\right|^{2}\left\|P_{2 n}\right\|^{2} \\
& \leq C \sum_{n} \frac{2}{n^{2}(2 n+1)} \leq C \sum_{n} \frac{1}{n^{3}}<+\infty,
\end{aligned}
$$

where we used that, for $(s+1) p \leq k \leq(s+1) p+p$

$$
\left|a_{2 k}\right| \leq \max _{j=0, \ldots, p}\left|A_{j}((p+1) s)\right| \leq \frac{C_{2}}{k},
$$

see Lemma 3.2, (3).

On the other hand, we have for $\psi_{e}^{\prime}$ by $(1.5)$

$$
\begin{aligned}
\psi^{\prime} & =\sum_{k=0}^{\infty} a_{2 k} P_{2 k}^{\prime}=\sum_{k=0}^{\infty} a_{2 k}\left(\sum_{j=1}^{k}(4 j-1) P_{2 j-1}\right) \\
& =\sum_{j=1}^{\infty}(4 j-1) P_{2 j-1}\left(\sum_{k=j}^{\infty} a_{2 k}\right) .
\end{aligned}
$$

Set, for brevity, $R_{j}=\sum_{k=j}^{\infty} a_{2 k}$. Recalling the definition of the sequence $\left\{a_{2 k}\right\}_{k}$ (3.15), (3.16), we have

$$
\sum_{k=(p+1) s}^{(p+1) s+p} a_{2 k}=0
$$

Consequently, for $k$ large enough $k=(p+1) s+r, 0 \leq r \leq p$, we get

$$
\sum_{j=k}^{\infty} a_{2 k}=\sum_{j=k}^{(p+1) s+p} a_{2 k}
$$

In particular, the properties stated in Lemma 3.2 say that

$$
\sum_{j=(p+1) s+1}^{(p+1) s+p} a_{2 k}=-A_{0}((p+1) s),
$$

and so

$$
\left|\sum_{j=(p+1) s+1}^{(p+1) s+p} a_{2 k}\right|=\left|A_{0}((p+1) s)\right| \geq \frac{C}{(p+1) s} .
$$


Therefore,

$$
\begin{aligned}
\left\|\psi_{e}^{\prime}\right\|_{L^{2}[-1,1]}^{2} & =\sum_{j=1}^{\infty}(4 j-1)^{2}\left\|P_{2 j-1}\right\|^{2}\left|R_{j}\right|^{2} \\
& \geq \sum_{s=N_{0}+1}^{\infty}(4(p+1) s+3)^{2}|| P_{2(p+1) s+1} \|^{2}\left|D_{(p+1) s+1}\right|^{2} \\
& \geq C \sum_{s=N_{0}+1}^{\infty} \frac{(4(p+1) s+3)}{((p+1) s)^{2}}=+\infty
\end{aligned}
$$

It remains to show that $T_{\psi} \in \mathcal{S}_{\delta_{0}}$. For this purpose, we have to bound $C_{2 n}(\psi)$ for $n$ large enough. Once again, write $n=(p+1) l+r, 0 \leq r \leq p$, and $l=[n /(p+1)]$. Recalling (3.2), we continue as

$$
\begin{aligned}
C_{2 n}(\psi) & =\sum_{k=0}^{n} d_{2 n, 2 k} a_{2 k}=\sum_{s=0}^{l-1}\left(\sum_{j=0}^{p} d_{2 n, 2((p+1) s+j)} a_{2((p+1) s+j)}\right) \\
& +\sum_{j=0}^{r} d_{2 n, 2((p+1) l+j)} a_{2((p+1) l+j)}=:(I)+(I I) .
\end{aligned}
$$

Since $d_{2 n, 2 k} \leq \exp \left(-C k^{2} / n\right)$, see (3.8), we easily obtain that $|(I I)| \leq e^{-C n}$. Reminding the definition of $\left\{a_{2 k}\right\}_{k}$ (3.15), (3.16), and relation (3.9) from Lemma 3.2, we come to

$$
\begin{aligned}
(I) & =\sum_{s=0}^{l-1}\left(\sum_{j=0}^{p} d_{2 n, 2((p+1) s+j)} a_{2((p+1) s+j)}\right) \\
& =\sum_{s=0}^{l-1}\left(\sum_{j=0}^{p} d_{2 n, 2((p+1) s+j)} A_{j}((p+1) s)\right) \\
& =\sum_{s=0}^{[n /(p+1)]-1} d_{2 n, 2(p+1) s} \prod_{j=1}^{p} \frac{1}{(2 n+2((p+1) s+j)+1)} .
\end{aligned}
$$

Take a small $0<\delta^{\prime}<1 / 2$ and cut the above sum in two pieces: the first sum is taken for $s=0, \ldots,\left[n^{1 / 2+\delta^{\prime}} /(p+1)\right]$, and second one is taken for $s=\left[n^{1 / 2+\delta^{\prime}} /(p+\right.$ $1)]+1, \ldots,[n /(p+1)]-1$. We have

$$
\begin{aligned}
\left|\sum_{s=0}^{\left[n^{1 / 2+\delta^{\prime}} /(p+1)\right]} \cdots\right| & \leq C \sum_{s=0}^{\left[n^{1 / 2+\delta^{\prime}} /(p+1)\right]} 1 \cdot \frac{1}{n^{p}} \\
& \leq \frac{n^{1 / 2+\delta^{\prime}}}{n^{p}}=\frac{1}{n^{p-1 / 2-\delta^{\prime}}}
\end{aligned}
$$

and, similarly,

$$
\begin{aligned}
\left|\sum_{s=\left[n^{\left.1 / 2+\delta^{\prime} /(p+1)\right]+1}\right.}^{[n /(p+1)]-1} \cdots\right| & \leq C \sum_{s=\left[n^{\left.1 / 2+\delta^{\prime} /(p+1)\right]+1}\right.}^{[n /(p+1)]-1} \frac{e^{-C s^{2} / n}}{n^{p}} \\
& \leq C \sum_{s=\left[n^{\left.1 / 2+\delta^{\prime} /(p+1)\right]+1}\right.}^{[n /(p+1)]-1} \frac{e^{-C n^{2 \delta^{\prime}}}}{n^{p}} \leq C_{\epsilon} e^{-C n^{2 \delta^{\prime}+\epsilon}}
\end{aligned}
$$

for any small $\epsilon>0$. Hence, we obtain the bound

$$
\left|C_{2 n}(\psi)\right| \leq \frac{C}{n^{p-1 / 2-\delta^{\prime}}} .
$$


Since $0<\delta^{\prime}<1 / 2$, it implies that $T_{\varphi} \in \mathcal{S}_{1 /(p-1)}$; by the choice of $p$ we have $1 /(p-1)<\delta_{0}$ and therefore $T_{\varphi} \in \mathcal{S}_{\delta_{0}}$. The theorem is proved.

Few remarks are in order. First, using the asymptotics of Legendre polynomials $\left\{P_{n}\right\}[5,3]$, one can show that the function $\varphi$ constructed in the above theorem, is in $L^{\infty}[0,1]$. Second, one can obviously get "a larger stock" of functions $\varphi$ with the properties claimed in Theorem 3.3. That is, instead of definitions (3.15), (3.16), one can define the coefficients $\left\{a_{2 k}\right\}_{k}$ as

$$
\begin{aligned}
& (3.17)\left(a_{2(p+1) s}, a_{2((p+1) s+1)}, \ldots a_{2((p+1) s+p)}\right) \\
& := \begin{cases}(0, \ldots, 0) & , \quad s<N_{0}, \\
((p+1) s)^{\delta}\left(A_{0}((p+1) s), A_{1}((p+1) s), \ldots A_{p}((p+1) s) \quad, \quad s \geq N_{0},\right.\end{cases}
\end{aligned}
$$

with $0 \leq \delta<2$. Indeed, the computations show that, in this case

$$
\begin{aligned}
\left\|\psi_{e}\right\|_{L^{2}[-1,1]}^{2} & \leq C \sum_{n} \frac{1}{n^{3-\delta}}, \quad\left\|\psi_{e}^{\prime}\right\|_{L^{2}[-1,1]}^{2} \geq C \sum_{n} \frac{1}{n^{1-2 \delta}}, \\
\left|C_{2 n}(\psi)\right| & \leq \frac{C}{n^{p-\left(1 / 2+\delta^{\prime}\right)(1+\delta)}} .
\end{aligned}
$$

Acknowledgments. We would like to thank L. Golinskii and S. Naboko for useful discussions on the subject of the article. B. Touré gratefully acknowledges the financial support coming from agreements between University of Bordeaux and the Embassy of France in Mali 2017, 2018.

\section{REFERENCES}

[1] Adams, R. Sobolev spaces. Pure and Applied Mathematics, Vol. 65. Academic Press, New York-London, 1975; exists also as: Adams, R.; Fournier, J. Sobolev spaces. Second edition. Pure and Applied Mathematics, Vol. 140. Elsevier/Academic Press, Amsterdam, 2003.

[2] Abramowitz, M.; Stegun, I. Handbook of mathematical functions with formulas, graphs, and mathematical tables. U.S. Government Printing Office, Washington D.C., 1964.

[3] Arfken, G.; Weber, H. Mathematical methods for physicists. Fifth edition. Harcourt/Academic Press, Burlington, MA, 2001.

[4] Axler, S. Bergman spaces and their operators, Surveys of some recent results in operator theory, I, Pitman Res. Not. Math. Ser. Vol. 171, 1988, p. 1-50.

[5] Erdélyi, A.; Magnus, W.; Oberhettinger, F.; Tricomi, F. Higher transcendental functions. Vol. II. Based on notes left by Harry Bateman. Reprint of the 1953 original. Robert E. Krieger Publishing Co., Inc., Melbourne, Fla., 1981.

[6] Hedenmalm, H.; Korenblum, B.; Zhu, K. Theory of Bergman spaces. Graduate Texts in Mathematics, 199. Springer-Verlag, New York, 2000.

[7] Gohberg, I.; Goldberg, S.; Kaashoek, M. Basic classes of linear operators. Birkhäuser Verlag, Basel, 2003.

[8] Grudsky, S.; Vasilevski, V. Bergman-Toeplitz operators: radial component influence. Integr. Eq. Oper. Theory 40 (2001), 16-33.

[9] Grudsky, S.; Karapetyants, A.; Vasilevski, N. Dynamics of properties of Toeplitz operators with radial symbols. Integr. Eq. Oper. Theory 50 (2004), no. 2, 217-253.

[10] Korenblum, B.; Zhu, K. An application of Tauberian theorems to Toeplitz operators. J. Operator Theory 33 (1995), no. 2, 353-361.

[11] Louhichi, I.; Zakariasy, L. On Toeplitz operators with quasi-homogeneous symbols. Arch. Math. (Basel) 85 (2005), no. 3, 248-257.

[12] Louhichi, I.; Strouse, E.; Zakariasy, L. Products of Toeplitz operators on the Bergman space. Integr. Eq. Oper. Theory 54 (2006), no. 4, 525-539.

[13] Louhichi, I.; Randriamahaleo, F.; Zakariasy, L. On the commutativity of a certain class of Toeplitz operators. Concr. Oper. 2 (2015), 1-7.

[14] Luecking, D. Forward and reverse Carleson inequalities for functions in Bergman spaces and their derivatives. Amer. J. Math. 107 (1985), no. 1, 85-111.

[15] Luecking, D. Trace ideal criteria for Toeplitz operators. J. Funct. Anal. 73 (1987), no. 2, $345-368$.

[16] Nikolski, N.. Operators, functions, and systems: an easy reading, I, II. AMS Mathematical Surveys and Monographs, Vols. 92, 93. AMS, Providence, RI, 2002. 
[17] R. Schmied, personal communication, 27/02/2005; see also http://mathworld.wolfram.com/LegendrePolynomial.html.

[18] Simon, B. Trace ideals and their applications. Second edition. Mathematical Surveys and Monographs, Vol. 120. AMS, Providence, RI, 2005

[19] Stroethoff, K. Compact Toeplitz operators on Bergman spaces. Math. Proc. Cambridge Philos. Soc. 124 (1998), no. 1, 151-160.

[20] Zorboska, N. The Berezin transform and radial operators. Proc. Amer. Math. Soc. 131 (2003), no. 3, 793-800 (electronic).

[21] Zhu, K. Operator theory in function spaces. Second edition. Mathematical Surveys and Monographs, 138. American Mathematical Society, Providence, RI, 2007.

[22] Zhu, K. Positive Toeplitz operators on weighted Bergman spaces of bounded symmetric domains. J. Operator Theory 20 (1988), no. 2, 329-357.

Faculté des Sciences, Université Amar Telidji - Laghouat, B.P. 37G, route de Ghardaia, Laghouat 03000, Algérie

E-mail address: zbendaoud@gmail.com

Institut de Mathématiques de Bordeaux UMR5251, CNRS, Université de Bordeaux, 351 ave. de la Libération, 33405 Talence Cedex, France

E-mail address: skupin@math.u-bordeaux.fr

Faculté des Sciences Exactes, des Sciences de la Nature et de la Vie, Université Mohamed Khider - Biskra, B.P. 145, Biskra 07000, Algérie

E-mail address: kamel_toumache@yahoo.fr

Institut Universitaire de Formation Professionnelle, Université de Ségou, B.P. 97, SÉGOU, MALI

Faculté des Sciences et des Techniques, Université des Sciences, des Techniques et des Technologies de Bamako, Campus Universitaire de Badalabougou À Bamako, B.P. E-3206, BAmAKO, MALI

E-mail address: vbelco@yahoo.fr

Institut de Mathématiques de Marseille, UMR 7373, Aix-Marseille Université, 39 rue F. Joliot Curie, 13453 Marseille Cedex 13, France

E-mail address: rzarouf@gmail.com 